\title{
Heat Conductivity of Three Periodic Hard Disks via Nonequilibrium Molecular Dynamics
}

\author{
William G. Hoover ${ }^{1,2}$ and Karl W. Kratky ${ }^{1}$
}

Received July 2, 1985; revision received October 28, 1985

\begin{abstract}
We use a driving field, of the type first suggested by Evans, to generate a steady heat current in the simplest possible system, a two-dimensional periodic "fluid" of three hard disks. Hard-disk motion equations can be conveniently derived from repulsive constant-force or linear-force potentials by considering the infinitely repulsive limit of these potentials. We show that the isoenergetic and isokinetic forms of the nonequilibrium equations of motion generate steady-state heat conductivities differing by terms of order $1 / N$, where $N$ is the number of particles. The resulting conductivities appear to vary as the logarithm of the driving field strength. Even at low fields, the three-body periodic-system results lie well below Enskog's infinite-system prediction.
\end{abstract}

KEY WORDS: Nonequilibrium molecular dynamics; heat conductivity; nonlinear transport; Gauss' principle of least constraint; hard disks.

\section{MOTIVATION AND INTRODUCTION}

Small systems can provide instructive simple models for complicated phenomena and tests for approximate theories. For instance, the "correlated-cell model" for melting describes the equilibrium "melting" of a periodic system of two hard disks or spheres. ${ }^{(1)}$ In the high-density phase each of the two particles is trapped in a cage composed of the periodic

\footnotetext{
${ }^{1}$ Institute for Experimental Physics, University of Vienna, Boltzmanngasse 5, Wien A-1090, Austria.

${ }^{2}$ Permanent address: Department of Applied Science, University of California at Davis-Livermore \& Lawrence Livermore National Laboratory, Post Office Box 808, Livermore, California 94550 .
} 
images of its "neighbor," the other particle. As the density is reduced it suddenly becomes possible for the mutually caged particles to "separate." Each can then percolate through a periodic crystal composed of the other's periodic images. This percolation can be described in either of two alternative ways: in the "infinite-space" view, the particle coordinates are allowed to grow without bound, and the two-particle force law depends on the interparticle separation in a periodic way; in the "finite-space" view, the particle coordinates are confined to a fixed cell and the interaction is short ranged. In the latter case a particle "leaving" the fundamental unit cell of the lattice is reintroduced, otherwise unchanged, on the opposite side of the cell.

This latter finite-space viewpoint is usual in discussions of computer simulations of small systems. It has the property of "mixing" the particles' spatial coordinates. As the calculation proceeds, the coordinates within the fundamental cell no longer contain explicit information as to the number of times the particles have traversed the periodic boundaries. In the equilibrium case this "mixing" has no important consequences.

We use the word "mixing" in a nontechnical sense (hence the quotation marks) to indicate that the information associated with the integers $n_{x}$ and $n_{y}$ distinguishing the various periodic images at coordinates $x+n_{x} L_{x}, y+n_{y} L_{y}$ is lost in a finite-space calculation.

Away from equilibrium the situation is different. Away from equilibrium, driving fields, performing work, and constraint forces, extracting heat, can be used to accelerate particles. In such cases the "external work," calculated by integrating the driving force times the particle displacements, depends upon the "infinite-space" displacements of the particles. The information associated with these displacements is destroyed by the periodic boundaries. The periodic boundaries introduce a spatial "mixing," enforcing long-range periodicity and homogeneity, even in the presence of accelerating directional fields.

By now, many simple nonequilibrium models of small periodic systems have been studied. Solutions of the Boltzmann equation for both diffusive and shear flows of two disks and two spheres have been obtained. ${ }^{(2-4)}$ In addition to the steady-state shear-thinning and normal stresses, ${ }^{(2,3)}$ the complete time-dependence of the transient flows, ${ }^{(3,4)}$ and the time development of the nonequilibrium entropy ${ }^{(3)}$ are now available.

In all of this new nonequilibrium work periodic boundaries have been used to obtain solutions that are spatially homogeneous and depend only slightly on the number of particles used. Unfortunately, existing theories were largely developed before computer simulation became widespread. These theories were formulated without explicitly taking the boundaries into account. For this reason, many of the nonequilibrium theoretical 
developments are not directly applicable to the systems studied using molecular dynamics. The nonequilibrium entropies and other properties resulting from the new solutions ${ }^{(2-4)}$ should provide useful guides to the generalization of the Zubarev-Jaynes nonlinear response theory ${ }^{(5,6)}$ to include the "mixing" effect inherent in the use of periodic boundaries.

An exact small-system analysis of heat flow is more complicated than the corresponding analyses for diffusion and viscosity. Diffusive and shear flows can be simulated with periodic two-body systems. Three bodies are required for the kinetic transfer of heat in the absence of a mass current. The corresponding three-body kinetic-theory calculations are similar in complexity to those required to estimate the magnitudes of the hydrodynamic "long-time" tails. We expect that a general awareness of these outstanding, tractable, and important, few-body problems in the kinetic theory of periodic systems will stimulate progress toward analytical solutions.

None of these nonequilibrium problems could have been posed precisely in the absence of an explicitly formulated "thermostat" designed to maintain constant energy or constant temperature. In the computer simulations it is usual to define the temperature in terms of the kinetic energy $K$. With this definition $-K=N k T$ in two dimensions, $3 N k T / 2$ in three dimensions - a special constraint force keeping the kinetic temperature constant can be formulated. Similar constraints can alternatively be applied to the total energy, $E=K+\Phi$, or to other thermodynamic and hydrodynamic variables.

Mechanics and ensemble theory have recently been used to suggest general methods for finding equations of motion incorporating thermostats or other constraints. Gauss gave a general prescription for constraint forces in his "principle of least constraint." (7) Nosé( ${ }^{(8,9)}$ suggested a more specialized alternative designed to reproduce the phase-space distributions associated with Gibbs' canonical and isobaric-isothermal ensembles. In addition to these two approaches, there are many other forms, ${ }^{(10)}$ suggested by control theory, which might prove useful.

From both the computational and theoretical points of view, it is desirable that the equations of motion both be time reversible and exhibit an energy-like constant of the motion. A system of equations giving the time-development of the particle coordinates is said to be time reversible if a movie of the motion, run backwards, still satisfies the same equations. Both Gauss' and Nosé's equations are time reversible. Both sets of equations also have energy-like constants of the motion. In the equilibrium case the constants correspond to the constrained variables or to a total energy. In the nonequilibrium case the constants are more complicated in form, but still correspond to effective energies. These constants and the 
feature of time reversibility facilitate comparisons and checks of the work. They also simplify analytic treatment. The equations of motion describing Gauss' isoenergetic and isothermal molecular dynamics are the simplest having these desirable characteristics. We therefore adopt them here.

For larger systems, Nosé's canonical thermostat ${ }^{(8-10)}$ is almost as useful. Both Gauss' constraint thermostat and Nosés canonical thermostat have equations of motion which fit nicely into the theoretical treatment of systems far from equilibrium being developed by Evans, Morriss, and Holian. ${ }^{(6,11)}$ Because the theory is now in a primitive state, lacking as it does an explicit treatment of the mixing effect of periodic boundaries, accurate small-system results should be valuable guides to the necessary generalizations.

In Section 2 we describe and discuss the differential equations of motion governing heat flow in Gaussian isoenergetic and isothermal (isokinetic) systems of three hard disks. Corresponding calculations of conductivity for many-body Lennard-Jones systems ${ }^{(12-14)}$ and for soft spheres $^{(15,16)}$ using the same ${ }^{(12,15,16)}$ or closely related ${ }^{(13,14)}$ methods have appeared during the past three years. In Section 3 we describe and discuss the resulting thermodynamic and hydrodynamic properties.

\section{ISOENERGETIC AND ISOKINETIC EQUATIONS OF MOTION FOR HARD DISKS}

We consider three classical hard disks, each with mass $m$ and diameter $\sigma$, confined to a square periodic box. The periodic boundaries eliminate edge effects. Such a system, with fixed center of mass and a Gaussian constraint force fixing either the total energy $E$ or the kinetic energy $K$, could be described in a seven-dimensional phase space spanned by four coordinates and three momenta. In Gaussian dynamics the thermodynamic friction coefficient $\zeta$, used to impose the constraint of constant $E$ or $K$, is an explicit function of the coordinates and momenta, not an independent variable.

The analogous system described with a Nosé canonical thermostat would require two more independent dynamical variables, so that the phase space would be nine-dimensional. In the Nosé case, four coordinates and four momenta, plus the thermodynamic friction coefficient $\zeta$, would be required. In the Nosé case the friction coefficient is not an explicit function of coordinates and momenta, but instead depends on the integrated time history of the system, very much as does a "memory function."

In either case, Gauss or Nosé, it is more convenient to work with the full set of all six space coordinates and all six momenta. The Gaussian constant-energy or constant-temperature constraint is incorporated directly 
into the equations of motion. The form of the constraint force follows from Gauss' principle of least constraint, and is linear in the momenta ${ }^{(7)}$ :

$$
F_{c}=-\zeta p=-\zeta m \dot{r}
$$

Each of the particles in the system has a constraint force proportional to its momentum, of the form (1), added to the other forces acting on it. The friction coefficient $\zeta$ in (1) varies with time so as to fix either the total energy, $E=\Phi+K$, or the kinetic energy $K$. In the most general case, the particle accelerations include three contributions in addition to those from the constraint force (1). These are the "applied" forces $F_{a}$ from the interparticle interactions, the "boundary" forces $F_{b}$, and the "driving" forces $F_{d}$. In our periodic simulations boundary forces are absent.

As is usual, we denote the "heat flux vector" by $Q$. The heat flux vector gives the magnitude and direction of the flow of energy, per unit time and area (length in two dimensions), averaged over the periodic volume $V$. $V$ is an area in our two-dimensional systems. The form of the heat flux is readily established ${ }^{(12-16)}$ :

$$
Q V=\sum \dot{r}_{i} E_{i}+\sum \sum\left(r_{i j}\right)\left[F_{i j} \cdot\left(\dot{r}_{i}+\dot{r}_{j}\right) / 2\right]
$$

where $E_{i}$ is the kinetic energy of particle $i$ plus half the potential energy particle $i$ shares with other particles $j$. The double sum includes each distinct pair of particles once.

To produce a heat flow consistent with linear response theory and with irreversible thermodynamics, we follow Evans, ${ }^{(12,13)}$ introducing the parameter $\lambda$ to indicate the relative strength of the "driving" force $F_{d}$. Each particle is accelerated by a force, proportional to $\lambda$, and depending upon that particle's contributions to the energy $E$ and to the potential part of the pressure tensor $P^{\varphi}$. The field-strength parameter $\lambda$ has units of inverse length. The product $\lambda T$ plays the role of the temperature gradient $\nabla T$ in driving a heat flux $Q$.

We use $\delta E$ and $\delta P^{\varphi}$ to indicate the energy and potential pressure contributions of a particle, relative to the instantaneous mean value - so that $\delta E_{1}=E_{1}-\left(E_{1}+E_{2}+E_{3}\right) / 3$, for instance. We arbitrarily choose to generate a heat flow parallel to the $x$ axis. With that choice, Evans' driving force is

$$
F_{d}=\lambda\left(\delta E+\delta P_{x x}^{\varphi} V, \delta P_{x y}^{\varphi} V\right)
$$

Knowing the driving force, we can calculate the Gaussian friction coef- 
ficient $\zeta$ in the constraint force (1) necessary to keep the total energy $E$ or kinetic energy $K$ constant:

$$
\begin{aligned}
& \zeta_{E}=\left(\lambda Q_{x} V\right) / 2 K \\
& \zeta_{K}=\left(\lambda Q_{x} V-\dot{\Phi}\right) / 2 K
\end{aligned}
$$

where $Q_{x}$ is the $x$ component of the heat flux vector $Q$.

The 12 equations of motion, four for each disk, have the following form:

$$
\begin{array}{ll}
\dot{x}=p_{x} / m, & \dot{p}_{x}=F_{x}-\zeta p_{x}+\lambda\left(\delta E+\delta P_{x x}^{\varphi} V\right) \\
\dot{y}=p_{y} / m, & \dot{p}_{y}=F_{y}-\zeta p_{y}+\lambda\left(\delta P_{x y}^{\varphi} V\right)
\end{array}
$$

where $\zeta$ is given by (4) and is the same for all of the particles. On the other hand, we emphasize that each particle has its own individual relative energy $\delta E$ and relative potential pressure tensor $\delta P^{\phi}$. The total momentum is a constant of the motion which we choose to set equal to zero. The applied forces $F$, constraint forces $-\zeta p$, and driving forces (proportional to $\lambda$ ) separately sum to zero, so that, from the "infinite-space" view, the center of mass is also fixed.

The interparticle forces $F_{x}$ and $F_{y}$ are calculated taking the periodic boundary into account, using the nearest-image convention. The "driving" force, proportional to $\lambda$, and the "constraint" or "control" force, proportional to $\zeta$, produce curved trajectories, even in the absence of interparticle forces.

It is easy to see that the equations of motion (5) are time reversible. The variables which change sign in the reversed motion include $\{\dot{x}, \dot{y}\},\left\{p_{x}, p_{y}\right\}$, and $\zeta$. The coordinates $\{x, y\}$, forces $\left\{F_{x}, F_{y}\right\}$, and $\lambda$ are unchanged in the reversed motion. Because the heat flux $Q$ changes sign in the reversed motion while the direction of the driving force does not, the equations exhibit the same paradoxical behavior as do Newton's equations: mathematical reversibility with the capability of simulating irreversible behavior. In any long-time-average finite-precision solution of the "reversible" equations of motion, one invariably finds a positive diffusion coefficient, positive viscosities, and a positive heat conductivity.

In the intervals between collisions, $F_{x}$ and $F_{y}$ vanish, and the isoenergetic and isokinetic trajectories coincide. In this degenerate situation, the motion equations (5) become relatively simple in structure, quartic functions of the momenta $\left\{p_{x}, p_{y}\right\}$. Even so, we were not able to integrate the free-flight motion equations analytically and resorted to numerical integration. 
In numerical work, it is convenient to integrate the equations (5) in two separate stages, using a "streaming" routine between collisions followed by a separate "collision" routine during collisions. The first routine calculates trajectories between collisions, when all of the interparticle forces $F_{x}$ and $F_{y}$ are zero. The second evaluates the momentum transfers that occur during collisions. During the collisions the equations can be simplified. The coordinates are kept fixed and the progress of the momenta $p_{x}$ and $p_{y}$ is followed under the influence of constant applied forces with time-varying constraint and driving forces.

In our exploratory work we used several forms and strengths of the interparticle forces to verify that the fixed-coordinate limit was well defined, and independent of the form of the force law. The force used in the work reported here, in Table I, with the collisions carried out at fixed coordinates, was a constant, and was applied to the colliding particles until the time integral of the colliding pair's relative momentum again reached its precollision value, zero. This constant-force collision model is equivalent to considering a steep effective repulsive potential, linear in the colliding pair's separation between $\sigma$ and $\sigma+\delta \sigma$, and taking the limit $\delta \sigma \rightarrow 0$.

In our calculations each collision had to be calculated twice, once with the isoenergetic equations of motion, (4E) and (5), and once with the isokinetic equations of motion, (4K) and (5). In each of these cases the coordinates were fixed and the driving force contribution $\lambda \delta E$ was set equal

Table I. Pressure and Heat Conductivity for Three Hard Disks as a Function of Field Strength $\lambda^{a}$

\begin{tabular}{ccccccc}
\hline & & & & \multicolumn{2}{c}{$\kappa(\sigma / k)(m / k T)^{1 / 2}$} \\
\cline { 5 - 7 }$n$ & $P_{x x} V / N k T$ & $P_{y y} V / N k T$ & $Q_{x} V(m / k T)^{1 / 2} / N k T$ & $(\mathrm{E})$ & $(\mathrm{K})$ \\
\hline 0 & $1.21+0.75,0.97$ & $0.79+0.65,0.83$ & $0.45+0.32,0.59$ & 0.22 & 0.30 \\
1 & $1.08+0.71,0.91$ & $0.92+0.70,0.91$ & $0.31+0.19,0.35$ & 0.29 & 0.38 \\
2 & $1.02+0.72,0.94$ & $0.98+0.74,0.98$ & $0.17+0.12,0.22$ & 0.33 & 0.46 \\
3 & $1.01+0.76,1.01$ & $0.99+0.74,0.98$ & $0.10+0.07,0.13$ & 0.39 & 0.54 \\
4 & $1.00+0.73,0.97$ & $1.00+0.75,1.00$ & $0.05+0.04,0.08$ & 0.42 & 0.59 \\
5 & $1.00+0.75,1.00$ & $1.00+0.74,0.98$ & $0.03+0.02,0.05$ & 0.45 & 0.68 \\
Eq & $1.00+0.74,0.99$ & $1.00+0.74,0.99$ & $0.00+0.00,0.00$ & & \\
\hline
\end{tabular}

a The volume $V=4 \sigma^{2}(3 / 4)^{1 / 2}$ is four times the close-packed volume. The dimensionless field strength $2 \sigma=(1 / 2)^{n}$ varied from $1(n=0)$ to $1 / 32(n=5)$. The streaming contributions to the pressure tensor and heat flux vector are given first, followed by the isoenergetic and isokinetic collisional contributions, separated by commas. The ealculations are based on runs of from 2500 to 15000 collisions. The uncertainties in $P V / N k T$ and $Q_{x} V(m / k T)^{1 / 2} / N k T$ are approximately 0.01 . The last line of Table I contains exact (analytic) equilibrium values. 
to zero. Both simplifications are exact for the limiting case in which the potential becomes a hard-disk potential. This too was verified by a series of calculations using repulsive potentials of various forms and strengths.

Because the rates of change of momenta and contributions to the pressure and to the heat flux vector differ, isoenergetic and isokinetic collisional averages had to be accumulated separately. We kept track of the time integrals of the streaming and collisional contributions to the pressure tensors and the heat flux vectors by adding the corresponding integrands to the 12 already being integrated in the motion equations (4) and (5).

It is interesting that even the time-averaged hard-disk collisional pressure tensors and heat-flux vectors for finite-system isoenergetic and isokinetic collisions differ. The reason for this difference can be understood by considering the effect of the constraint force $-\zeta p$. In the isokinetic case this force resists the slowing-down of the colliding pair's relative momentum. This leads to a "longer" (but still only infinitesimal) collision duration, with a correspondingly greater collisional momentum flux.

Averaged over impact parameters, the increased collision times in the isokinetic case increase the nonideal part of the thermodynamic pressure. ${ }^{(17)}$ In two dimensions the increase corresponds to a factor of $[N-1] /[N-(3 / 2)]$. Thus, in the large-system "thermodynamic limit" the two pressures coincide. The maximum difference between them occurs for the two-particle case. In that case the isokinetic collisional pressure is twice the isoenergetic value. For our three-disk system the isokinetic collisional pressure is predicted to exceed the corresponding isoenergetic contribution by a factor of $[3-1] /[3-(3 / 2)]=4 / 3$. This prediction is fully consistent with our numerical calculations. See Table I.

The isokinetic collisional heat fluxes are rather close to twice the corresponding isoenergetic fluxes, as is also shown in Table I. From the standpoint of Green-Kubo equilibrium fluctuation theory these nonequilibrium steady-state collisional fluxes include not only a "potential" contribution, but also a "cross" contribution, coupling the convective streaming with the impulsive collisions. These terms can be estimated relatively accurately, as was done for large systems by Alder, Gass, and Wainwright. ${ }^{(18)}$ From their work we expect that the heat-flux ratio depends upon the density as well as the strength of the driving field $\lambda$.

To simplify checking our work we chose a density, one fourth the close-packed density, for which the canonical partition function (and also the isoenergetic and isokinetic pressures) could be calculated analytically. The effects of order $1 / N$ relating molecular dynamics results to canonicalensemble averages have been considered before, by many workers. ${ }^{(17-20)}$ We avoided lower densities than one-fourth the close-packed density because, at strong fields, the equations of motion trap the particles in a 
streaming motion parallel to the field. This effect is absent at higher densities or in larger systems.

The isoenergetic and isokinetic collisions can be treated by (relatively complicated, but computationally less time consuming) analytic methods, which we are still developing and testing. We expect to report on these methods, applied to larger systems, in the future. ${ }^{(17)}$

\section{HARD-DISK CONDUCTIVITIES}

The three-disk isoenergetic and isokinetic conductivities found from the relation

$$
\kappa=Q_{x} / \lambda T
$$

are listed in Table I. We use the definition $T=K / 3 k$ for the temperature $T$. The maximum possible value of $Q_{x} V$, achieved when one particle has an $x$ velocity component $2(k T / m)^{1 / 2}$ and the others have $-(k T / m)^{1 / 2}$, is $3 m(k T / m)^{3 / 2}$.

For field strengths $\lambda$ greater than unity the three disks eventually achieve this free-streaming state, with the maximum collisionless value of $Q_{x} V$. This instability is not important at low field strengths. In the lowfield limit, Evans' analysis establishes that the heat current approaches the linear-response result $Q_{x}=\kappa \lambda T$. We use this same relation to define the heat conductivity $\kappa$ for finite $\lambda$. At finite fields the heat conductivity decreases below the apparent low-field limiting behavior.

At low fields the steady-state conductivity is obscured by statistical fluctuations. For three disks at the density we chose, one-fourth the closepacked density, there is a useful range of driving fields varying from $\hat{\lambda} \sigma=1$ to $1 / 32$. We gathered pressure and heat-flux data over runs of a few thousand collisions. The results in the Table suggest a logarithmic dependence of all three conductivity components (streaming, collisional isoenergetic, and collisional isokinetic) on field strength:

$$
\begin{aligned}
& \kappa(\sigma / k)(m / k T)^{1 / 2} \doteq 0.236+0.046 n \\
& \kappa(\sigma / k)(m / k T)^{1 / 2} \doteq 0.305+0.075 n
\end{aligned}
$$

where the dimensionless field strength $\lambda \sigma$ is $(1 / 2)^{n}$. These rough fits are compared to the data in Fig. 1.

We include also in Table I the measured pressure-tensor components, for comparison with those predicted from the canonical partition-function value for three disks, $(P V / N k T)_{\text {can }}=1.495$ at zero field. The prediction, giving $\left((P V / N k T)_{\mathrm{md}}=1.7425\right.$ for the isoenergetic dynamics and 1.9900 for the 


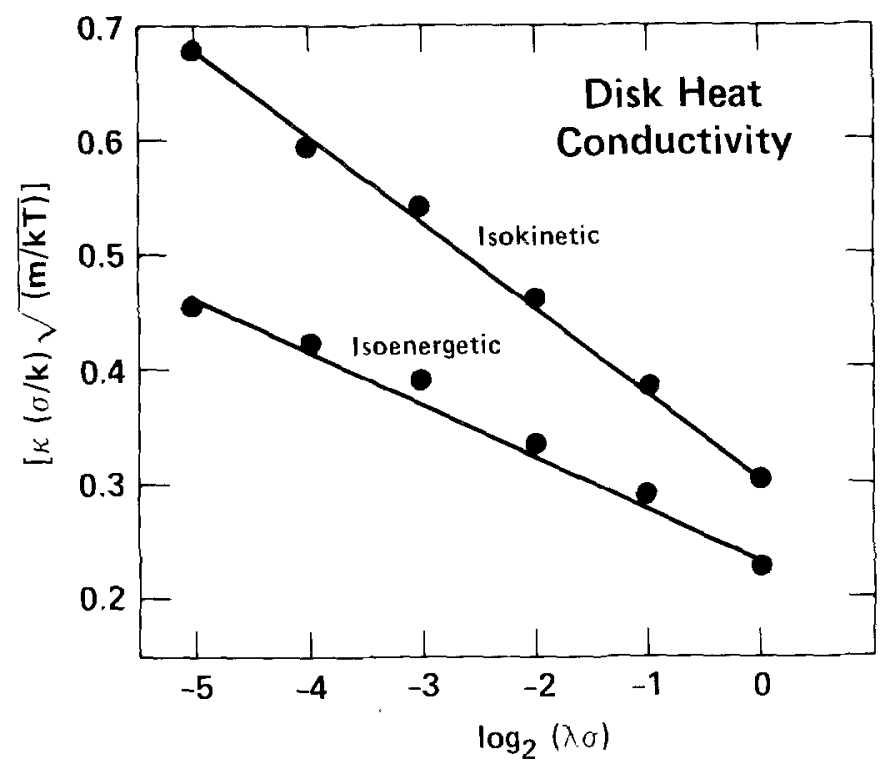

Fig. 1. Heat conductivity of three periodic hard disks. The data from Table I are shown along with the isoenergetic and isothermal fits given by Eq. (7) of the text.

isokinetic dynamics, is based on relations linking the canonical ensemble pressure to the molecular dynamics pressure through the pair distribution function. ${ }^{(17,19)}$ The isoenergetic case is independent of dimensionality:

$$
(N-1)(P V-N k T)_{\mathrm{md}}=N(P V-N k T)_{\mathrm{can}}
$$

The isokinetic case ${ }^{(17)}$ is more complicated. In two dimensions, the isokinetic pressure, for a fluid, has the form

$$
\left(N-\frac{3}{2}\right)(P V-N k T)_{\mathrm{md}}=N(P V-N k T)_{\mathrm{can}}
$$

Gass' Enskog-theory conductivity ${ }^{(21)}$-in the thermodynamic limit, for which the isoenergetic and isokinetic values coincide-provides an orderof-magnitude estimate for the thermodynamic state we investigated:

$$
\kappa(\sigma / k)(m / k T)^{1 / 2} \doteq 1.7
$$

The three-disk conductivities in Table I are all significantly less than the Enskog estimate and varied by roughly a factor of 2 over the useful range of field strengths. Preliminary results for 12 disks, at the same reduced density, lie close to Enskog's estimate (9). Kirkpatrick's success ${ }^{(22)}$ in explaining the shear-thinning found in computer experiments simulating viscous 
flows provides hope that the field dependence found here can be similarly understood. The form of the logarithmic dependence is the same as that found by Evans ${ }^{(23)}$ in a two-dimensional viscosity study. It is most interesting that this behavior already seems to appear in a three-body system.

\section{ACKNOWLEDGMENTS}

The continuous exchange of correspondence and preprints with Drs. Denis Evans, Gary Morriss, Brad Holian, and Prof. Gianni Iacucci has proved helpful in understanding the importance of small-system calculations to the development of a nonequilibrium theory. We thank Professor Dr. Peter Weinzierl too for his enthusiastic interest in and support of this work. Mag. Peter Karlsreiter was particularly helpful in arranging for the use of the VAX 11/750 (with floating point accelerator) computer at the Institute for Experimental Physics of the University of Vienna. W. G. H. is indebted to the Universities of California and Vienna as well as to the Lawrence Livermore National Laboratory and the National Science Foundation for financial support. The secretarial staff of the Institute provided us with an electric typewriter.

\section{REFERENCES}

1. B. J. Alder, W. G. Hoover, and T. E. Wainwright, Phys. Rev. Lett. 11:241 (1963).

2. A. J. C. Ladd and W. G. Hoover, J. Stat. Phys. 38:973 (1985).

3. G. P. Morriss, Phys. Lett. A (submitted).

4. W. G. Hoover, J. Stat. Phys. $42: 587$ (1986).

5. D. N. Zubarev, Nonequilibrium Statistical Thermodynamics (Consultants Bureau, New York, 1974), Section 27.

6. G. P. Morriss and D. J. Evans, Mol. Phys. 54:629 (1985).

7. D. J. Evans, W. G. Hoover, B. H. Failor, B. Moran, and A. J. C. Ladd, Phys. Rev. A 28:1016(1983).

8. S. Nosé, J. Chem. Phys. 81:511 (1984).

9. S. Nosé, Mol. Phys. 52:255 (1984).

10. D. J. Evans and W. G. Hoover, Ann. Rev. Fluid Mech. 18:243 (1986).

11. B. L. Holian, Phys. Rev. A (to appear, February 1986).

12. D. J. Evans, Phys. Lett. 91A:457 (1982).

13. M. Dixon and M. J. Gillan, J. Phys. C 16:869 (1983).

14. C. Massobrio and G. Ciccotti, Phys. Rev. A 30:3191 (1984).

15. W. G. Hoover, B. Moran, and J. Haile, J. Stat. Phys. 37:109 (1983).

16. R. Grover, W. G. Hoover, and B. Moran, J. Chem. Phys. 83:1255 (1985).

17. K. W. Kratky and W. G. Hoover (in preparation).

18. B. J. Alder, D. M. Gass, and T. E. Wainwright, J. Chem. Phys. 53:3813 (1970). 
19. W. G. Hoover and B. J. Alder, J. Chem. Phys. 46:686 (1967).

20. J. J. Erpenbeck and W. W. Wood, Molecular dynamics techniques for hard-core systems, in Statistical Mechanics, B. J. Berne, ed. (Plenum, New York, 1977), Part B.

21. D. M. Gass, J. Chem. Phys. 54:1898 (1971).

22. T. R. Kirkpatrick, Phys. Rev. Lett. 53:1735 (1984).

23. D. J. Evans, Phys. Rev. A 22:290 (1980). 